\title{
Shape-Selective Synthesis of Palladium Nanoparticles Stabilized by Highly Branched Amphiphilic Polymers**
}

\author{
By Ulf Schlotterbeck, Cyril Aymonier, Ralf Thomann, Herbert Hofmeister, Moniek Tromp, \\ Walter Richtering, and Stefan Mecking*
}

Despite the current broad interest in such materials, the synthesis of defined structures in the size range of $10 \mathrm{~nm}$ to ca. $1 \mu \mathrm{m}$ ("mesoscopic") is challenging. Few routes shape selectively afford geometrically regular structures, other than the typical spherical metal particles of 1 to $10 \mathrm{~nm}$. Moreover, these few routes are largely restricted to aqueous systems, however, for catal ysis and other applications dispersions in organic solvents are desirable. Carbon monoxide reduction of a palladium(II) com pound in combination with stabilization by (readily available) amphiphilic hyperbranched polymers surprisingly affords disper sions of hexagonal platelets selectively with average sizes of thirty to several hundred nanometers in toluene. The size can be controlled by the polymer composition. Transmission electron microscopy (TEM), electron diffraction, and extended X ray ab sorption fine structure (EXAFS) spectroscopy demonstrate these palladium(0) platelets to be extremely thin (1 $2 \mathrm{~nm})$. Despite this high aspect ratio, the platelets prove quite shear resistant.

\section{Introduction}

Particulate structures with sizes of $1 \mu \mathrm{m}$ down to about $1 \mathrm{~nm}$, i.e., the colloidal range, are becoming of increasing inter est. At the lower end of this size scale $(110 \mathrm{~nm})$ the transition from bulk properties to those of molecules occurs. Such small

[*] Prof. S. Mecking

Universität Konstanz, Fachbereich Chemie

Universitätsstr. 10, D-78457 Konstanz (Germany)

E-mail: stefan.mecking@uni-konstanz.de

Dr. U. Schlotterbeck, Dr. C. Aymonier, Dr. R. Thomann

Institut für Makromolekulare Chemie und

Freiburger Materialforschungszentrum

der Albert-Ludwigs-Universität Freiburg

Stefan-Meier-Str. 31, D-79104 Freiburg (Germany)

Dr. H. Hofmeister

Max-Planck-Institut für Mikrostrukturphysik

Weinberg 2, D-06120 Halle (Germany)

Dr. M. Tromp

Department of Inorganic Chemistry and Catalysis

Debye Institute, Utrecht University, NRSC-Catalysis

Sorbonnelaan 16, P.O. Box 80083

NL-3508 TB Utrecht (The Netherlands)

Prof. W. Richtering

RWTH Aachen, Institut für Physikalische Chemie

Templergraben 59, D-52056 Aachen (Germany)

[**] Financial support by the Volkswagen Foundation (Schwerpunkt Komplexe Materialien) is gratefully acknowledged. We thank the DFC (SFB428) and the Eugen Graetz Foundation for financial support. U. S. is grateful for a Baden-Württemberg Landesgraduierten stipend. S. M. is indebted to the Fonds der Chemischen Industrie and the Hermann-Schnell Foundation for financial support. A generous loan of noble metal salts by Umicore AG \& Co. KG is gratefully acknowledged. We thank $\mathrm{H}$. Kautz for a sample of linear polyglycerol. Discussions with C. Röhr and E. Schweda on the electron diffraction measurements are gratefully acknowledged by U. S. We thank the scientific staff of beamline X1.1 of the HASYLAB synchrotron (I-01-032 EC) for help with the EXAFS measurements. Supporting Information is available online from Wiley InterScience or from the author. particles of semiconductors or metals are of interest, e.g., for optoelectronics or catalysis. ${ }^{[1]}$ Slightly larger structures (beyond ca. $10 \mathrm{~nm}$ ) have been designated the rather general term "me soscopic". Such "mesoscopic" systems are of interest, e.g., for potential applications as functional surfaces.

The synthesis of metal nanoparticles and their properties have attracted considerable attention for more than a cen tury. ${ }^{[2]}$ They may be prepared by various synthetic routes, such as reduction of metal salts in the presence of suitable low mo lecular weight or polymeric stabilizers, ${ }^{[2]}$ electrochemical prep aration, ${ }^{[3]}$ decomposition of organometallic precursors, ${ }^{[4]}$ or va por deposition methods. ${ }^{[5]}$ Most often, spherical particles with sizes in the range of 1 to $10 \mathrm{~nm}$ are obtained. However, it has long been known that geometrically regular particles can also be obtained under suitable conditions. ${ }^{[6]}$ Despite recent ad vances towards an understanding of the factors controlling par ticle shape, particularly with respect to the effect of stabilizers on selective crystal growth, ${ }^{[7]}$ successful shape controlled parti cle synthesis is somewhat empirical to date. An early example of the synthesis of geometrically regular palladium nanoparti cles was reported by Turkevich and Kim, who described the formation of a mixture of trigonal, pentagonal, hexagonal, and other palladium particles by formate reduction with sodium polyacrylate as a stabilizer. ${ }^{[6 \mathrm{~d}]}$ Such particles can also be pre pared selectively, assisted by polymers ${ }^{[6 \mathrm{e}]}$ or low molecular weight stabilizers. ${ }^{[7 e-g]}$ Studies on the shape selective synthesis of metal nanoparticles with defined shapes have focused on particles smaller than $20 \mathrm{~nm}$. Only a few examples of the syn thesis of larger, geometrically regular particles with sizes of $20 \mathrm{~nm}$ to approximately $100 \mathrm{~nm}$, particularly with high aspect ratios, by convenient methods exist. ${ }^{[8]}$

The majority of work on nanoparticles with a defined shape has focused on aqueous systems. ${ }^{[9]}$ For catalysis of organic reac tions and other applications, stable solutions of metal nanopar ticles in apolar, inert organic solvents are desirable. 


\section{Results and Discussion}

Amphiphilic polymers with a hyperbranched, polar scaffold and an apolar periphery efficiently stabilize metal nanoparti cles in apolar, inert organic solvents. ${ }^{[10-12]}$ The polar, flexible scaffold interacts with the metal particle surface; the apolar moieties provide solubility in organic solvents, such as toluene. Hyperbranched polymers ${ }^{[13]}$ are randomly branched (degree of branching, ${ }^{[14]} \mathrm{DB}$, ca. $60 \%$ ) in comparison to dendrimers, ${ }^{[15]}$ which ideally possess a perfectly branched structure $(\mathrm{DB}=100 \%)$. Similar to dendrimers, the highly branched na ture of hyperbranched polymers results in a low crystallinity, a low viscosity, and a high solubility in many solvents. Moreover they possess a large number of functional end groups. Albeit that they are not monodisperse, hyperbranched polyethylene imine and polyglycerol can be prepared with a narrow molecu lar weight distribution (weight average molecular weight/num ber average molecular weight, $\left.M_{\mathrm{w}} / M_{\mathrm{n}}<1.5\right)$ in a single step protocol and are available commercially. ${ }^{[16-17]}$

For the present study, amphiphilic polymers obtained by par tial esterification with fatty acids of a polyglycerol core $(\mathrm{DB}=61 \%),{ }^{[18]}$ or by amidation of polyethyleneimine (DB $=60 \%)$, respectively, were employed. ${ }^{[11,19]}$ In both cases amphiphilic molecules result, which have no tendency for ex tensive aggregation in toluene solution, as evidenced by small angle neutron scattering (SANS, radius of gyration, $R_{\mathrm{g}}<5 \mathrm{~nm}$ for $1 \%$ toluene solutions). ${ }^{[10 \mathrm{~b}, 11,20]}$ Amphiphilic polyglycerols based on different core molecular weights ranging from $M_{\mathrm{n}}=2000 \mathrm{~g} \mathrm{~mol}^{-1}$ to $M_{\mathrm{n}}=10000 \mathrm{~g} \mathrm{~mol}^{-1}$, partially esterified (22 to $100 \%$ ) with two different hydrocarbon substituents $\mathrm{OC}(=\mathrm{O}) \mathrm{R}(\mathrm{R}=$ Pentadecyl $(\mathrm{C} 15)$ or $p$ Tolyl $(\mathrm{T}))$, were investi gated (designated as, e.g., PG2000 $\mathrm{T}_{0.52}$ for a polymer based on a $M_{\mathrm{n}}=2000 \mathrm{~g} \mathrm{~mol}^{-1}$ polyglycerol core, $52 \%$ of all $\mathrm{OH}$ groups esterified with tolyl groups). An amphiphilic polyethyleneim ine amide based on a core of molecular weight $M_{\mathrm{n}}=4000 \mathrm{~g} \mathrm{~mol}^{-1}$ amidated to $47 \%{ }^{[21]}$ with palmitic acid (PEI4000 C15 0.47 ) was also studied.

Reduction of $\mathrm{Pd}(\mathrm{OAc})_{2}$ in toluene solutions of the amphi philic polymers, with $\mathrm{H}_{2}$, with $\mathrm{LiBEt}_{3} \mathrm{H}$ ("superhydride") for the polyethyleneimine amides, or by the remaining $\mathrm{OH}$ groups of amphiphilic polyglycerol without added reducing agent at $60{ }^{\circ} \mathrm{C}$ afforded spherical palladium nanoparticles with sizes in the range of 1 to $9 \mathrm{~nm}$. Somewhat surprisingly, reduction with carbon monoxide resulted in stable toluene solutions of en tirely different, much larger geometrically regular particles (Fig. 1).

Under suitable conditions, hexagonal platelets with a rela tively narrow size distribution and reproducible extensions are formed exclusively (Table 1). The size of these platelets, i.e., the size of their hexagonal faces as defined in the Experimental section, is influenced by various parameters during their syn thesis.

For the amphiphilic polyglycerols, the nature of the lipophilic moiety $\mathrm{R}$ only has a minor influence on particle shape and size (Table 1, entries 8 to 11 compared to entries 4,5), whereas a higher degree of esterification results in larger platelets (en

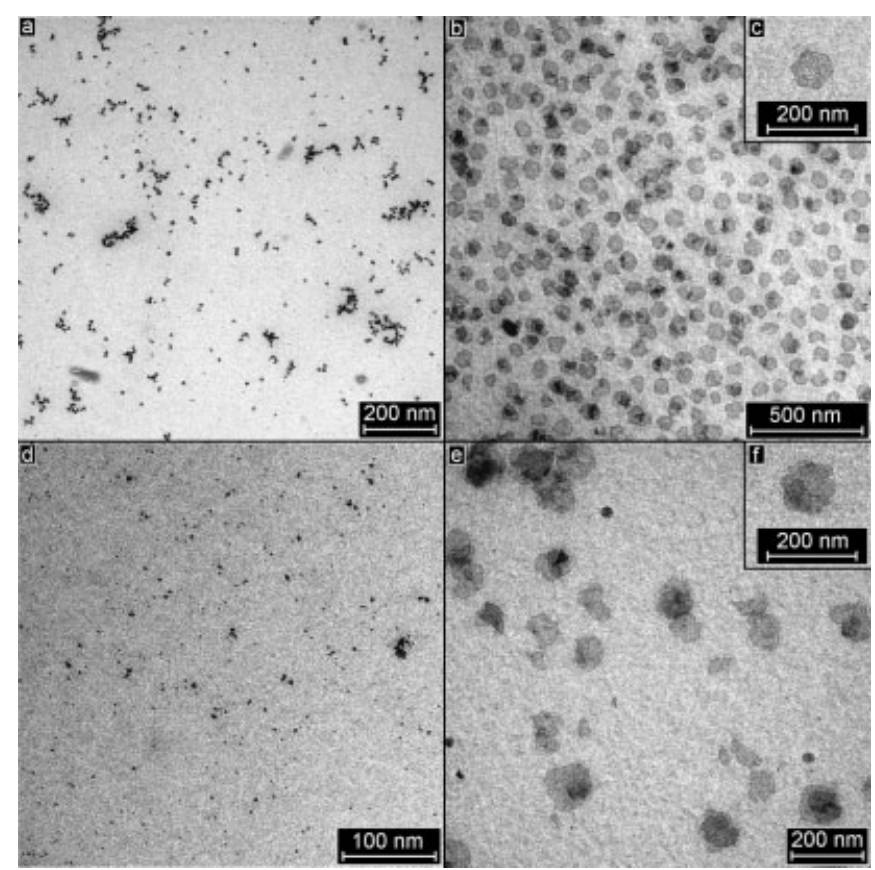

Figure 1. TEM images of palladium particles formed upon a) $\mathrm{H}_{2}$, b,c) $\mathrm{CO}$ reduction of $\mathrm{Pd}(\mathrm{OAc})_{2}$ in the presence of $\mathrm{PG} 4000-\mathrm{T}_{0.62}$, and d) $\mathrm{H}_{2}$, $\mathrm{e}, \mathrm{f}) \mathrm{CO}$ reduction of $\mathrm{Pd}(\mathrm{OAc})_{2}$ in the presence of $\mathrm{PEI} 4000-\mathrm{C} 15_{0.47}$.

Table 1. Particle synthesis: conditions and sizes of the hexagonal particles formed. Particle synthesis by exposing a solution of the amphiphilic polymer and $\mathrm{Pd}(\mathrm{OAc})_{2}$ to $\mathrm{CO}(1 \mathrm{~atm})$ at room temperature overnight. Particle size as defined in the Experimental section.

\begin{tabular}{|c|c|c|c|c|}
\hline No. & Polymer & $\begin{array}{c}\text { Polymer conc. } \\
{\left[\mathrm{g} \mathrm{L}^{\mathrm{l}}\right]}\end{array}$ & $\begin{array}{c}\text { Ratio of } \\
\text { polymer } / \mathrm{Pd}(\mathrm{OAc})_{2}\end{array}$ & $\begin{array}{c}\text { Particle size } \\
{[\mathrm{nm}]}\end{array}$ \\
\hline 1 & PEI4000-C15 0.47 & 1.0 & 0.33 & $72 \pm 28$ \\
\hline 2 & $\mathrm{PG} 2000-\mathrm{C} 15_{0.44}$ & 10.1 & 10.1 & $119 \pm 18$ \\
\hline 3 & PG $6000-C 15_{0.22}$ & 10.3 & 10.2 & $19 \pm 7$ \\
\hline 4 & PG $6000-\mathrm{C} 15_{0.48}$ & 10.4 & 10.3 & $30 \pm 14$ \\
\hline 5 & PG6000-C15 0.77 & 10.6 & 10.6 & $73 \pm 18$ \\
\hline 6 & PG6000-C15 1.00 & 10.3 & 10.2 & ca. 400 [a] \\
\hline 7 & $\mathrm{PG} 10000-\mathrm{C} 15_{0.65}$ & 9.9 & 9.6 & $303 \pm 48$ \\
\hline 8 & PG4000-T 0.62 & 10.0 & 18.8 & $54 \pm 16$ \\
\hline 9 & $P G 4000-T_{0.62}$ & 16.6 & 28.8 & $59 \pm 16$ \\
\hline 10 & $\mathrm{PG} 4000-\mathrm{T}_{0.62}$ & 16.5 & 19.1 & $58 \pm 16$ \\
\hline 11 & PG4000-T 0.62 & 17.8 & 10.3 & $57 \pm 12[\mathrm{~b}]$ \\
\hline 12 & PG $6000-C 15_{0.48}$ & 10.0 & 9.9 & $76 \pm 20\left(\right.$ at $\left.15^{\circ} \mathrm{C}\right)$ \\
\hline 13 & PG $6000-C 15_{0.48}$ & 10.0 & 9.9 & $32 \pm 12\left(\right.$ at $\left.25^{\circ} \mathrm{C}\right)$ \\
\hline 14 & PG $6000-C 15_{0.48}$ & 10.0 & 9.9 & $33 \pm 11$ (at $40^{\circ} \mathrm{C}$ ) [c] \\
\hline 15 & PG $6000-C 15_{0.48}$ & 10.0 & 9.9 & $46 \pm 14\left(\right.$ at $25^{\circ} \mathrm{C}$ ) [d] \\
\hline
\end{tabular}

[a] Largely aggregated. [b] Squares and triangles of up to $10 \mathrm{~nm}$ also observed [7e]. [c] Spherical particles also formed, presumably due to the beginning of reduction by the residual $\mathrm{OH}$ groups of the polyglycerol. [d] Sample shaken during reduction.

tries 3 6). With a completely esterified polyglycerol, platelets of up to ca. $500 \mathrm{~nm}$ are observed.

The molecular weight of the polyglycerol scaffold strongly influences the size of the platelets, albeit not in a simple sys tematic fashion. Whereas colloids prepared with PG2000 
$\mathrm{C} 15_{0.44}$ have sizes of around $100 \mathrm{~nm}$ (entry 2), the size is low ered to $30 \mathrm{~nm}$ for colloids generated in the presence of PG6000 C15 0.48 (entry 4). It can be speculated that the palla dium particle formation process is influenced by the flexibility of the scaffold ${ }^{[22]}$ as well as the size of the polymer molecules, which both increase with molecular weight. Applying polymers with a core molecular weight of $10000 \mathrm{~g} \mathrm{~mol}^{-1}$ results in much larger platelets (entry 7). Experiments with the amphiphilic polyglycerol PG4000 $\mathrm{T}_{0.62}$ reveal that the sizes of the resulting particles are not very sensitive towards the metal to polymer ratio or polymer concentration in the range examined (entry 8 to 11), which supports the assumption that the observed influ ence of the polymer molecular weight and degree of esterifica tion can indeed be traced back to these parameters.

Particles prepared by $\mathrm{CO}$ reduction in the presence of am phiphilic polyethyleneimine (PEI) are also hexagons, albeit less regular in comparison to the polyglycerol stabilized platelets. This demonstrates that platelet formation is not unique to the polyether polyol scaffold of the amphiphilic polyglycerols.

The reaction temperature had a strong influence on the re sults of the platelet synthesis: the platelet size decreased from 76 to $32 \mathrm{~nm}$ when the temperature was increased from $15^{\circ} \mathrm{C}$ to $25^{\circ} \mathrm{C}$. Raising the temperature to $40^{\circ} \mathrm{C}$ did not alter the plate let size further (entries 12 to 14), but additionally resulted in formation of some strongly aggregated structures, presumably due to the beginning of reduction of $\mathrm{Pd}(\mathrm{OAc})_{2}$ by the residual $\mathrm{OH}$ groups of polyglycerol.

Reduction with $\mathrm{CO}$ in the presence of a partially esterified linear analog of polyglycerol ${ }^{[18 \mathrm{~b}]}\left(M_{\mathrm{n}}=1500 \mathrm{~g} \mathrm{~mol}^{-1}\right.$, DB $0 \%$; esterified to $29 \%$ or $54 \%$ with $\mathrm{R}=\mathrm{C}_{15} \mathrm{H}_{31}$ ) resulted in precipi tation of palladium; no stable colloid was obtained. Polyvinyl pyrollidone (PVP) has been investigated thoroughly as a stabi lizer for metal colloids. ${ }^{[23]}$ Reduction of $\mathrm{Pd}(\mathrm{OAc})_{2}$ with $\mathrm{CO}$ in the presence of PVP in $\mathrm{CHCl}_{3}$ solvent resulted in the forma tion of irregular platelets and also spherical particles of ca. $2 \mathrm{~nm}$ diameter. Apparently the highly branched flexible poly mer scaffold in combination with the amphiphilic nature of the polymer is beneficial for regular platelet stabilization.

However, we believe that the structure and nature of the polymer are not the sole keys to shape selectivity, but rather the specific reduction process. For comparison, colloid forma tion by ligand displacement ${ }^{[4 \mathrm{c}]}$ from the palladium( 0 ) precursor $\left[\mathrm{Pd}(\mathrm{dba})_{2}\right](\mathrm{dba}=1,5$ diphenylpenta 1,4 dien 3 one $)$ with car bon monoxide was investigated. Spherical particles of 4 to $6 \mathrm{~nm}$ average size are obtained. The colloids are formed within seconds, whereas most of the aforementioned reduction meth ods occur much more slowly, as evidenced by the gradual darkening of the reaction solutions. Reduction with $\mathrm{LiBEt}_{3} \mathrm{H}$ also occurs within seconds; reduction with $\mathrm{CO}$ (resulting in hexagonal platelets) requires several hours; and reduction with hydro gen or reduction by the $\mathrm{OH}$ groups of polyglycerol without added reducing agent occur overnight. As a general rule, in the formation of any type of particles by reduction processes the rate of reduc tion will usually strongly affect particle nucleation and growth, ${ }^{[6]}$ and thus particle size and possibly also shape. With respect to reduction rate, the $\mathrm{CO}$ reduction does not stand out from other reductions that yield spherical particles. Rather, the observed unusual shape selectivity is specific to the reduction process of $\mathrm{Pd}^{\mathrm{II}}$ with $\mathrm{CO}$, in combination with a polymer that apparently adsorbs to certain crystal faces effectively and thus promotes specific crystal growth, as has been suggested for other types of colloid synthesis. ${ }^{[7 \mathrm{~h}, \mathrm{i}, 9 \mathrm{~d}]}$ It can be speculated that intermediate unstable palladium carbonyl compounds are in volved. In the final particles, the polyglycerol is adsorbed by means of its polar, flexible scaffold and the lipophilic moieties provide solubility in apolar media (Fig. 2).

Shape and structure of the platelets were further investigated by transmission electron microscopy (TEM). Tilting the sample in the microscope up to the technical limit of $60^{\circ}$, no indication of a significant extension of the nanoplatelets in the third di mension was observed. The thickness of the hexagonal plates was estimated by TEM of mixtures with separately prepared colloids composed of spherical particles of different sizes. For a mixture with spherical particles of $2 \mathrm{~nm}$ average diameter, the electron density of the platelets which lay flat on the grid ap peared even slightly lower in comparison to the spherical parti cles. This already indicates that the platelets are approximately 1 to $2 \mathrm{~nm}$ thick, corresponding to approximately five to ten layers of atoms.

Clear proof is provided by TEM on a sample, in which some of the platelets have arranged into stacks perpendicular to the grid (see Supporting Information). A platelet thickness of $1.3 \mathrm{~nm}$ is observed. Incidentally, it can also be noted that the distance of $5 \mathrm{~nm}$ between two platelets is in agreement with a polymer monolayer on each platelet, comparing the distance between platelets with the radius of gyration observed by SANS in solution. ${ }^{[20]}$

The platelet structure and composition were studied further by high resolution transmission electron microscopy (HRTEM). The relatively high electron beam intensity of the instrument setup used, which exceeds that of regular TEM in vestigations by two orders of magnitude, unfortunately results in disintegration of the hexagonal platelets to smaller frag ments. It can be speculated that the strong electron radiation decomposes the polymer and thus results in a "dewetting" of the particle surface, which induces fragmentation. Notwith standing, the fragments exhibit lattice plane fringes indicating the crystalline nature of these platelets, even after electron beam induced degradation (Fig. 3). The most commonly ob

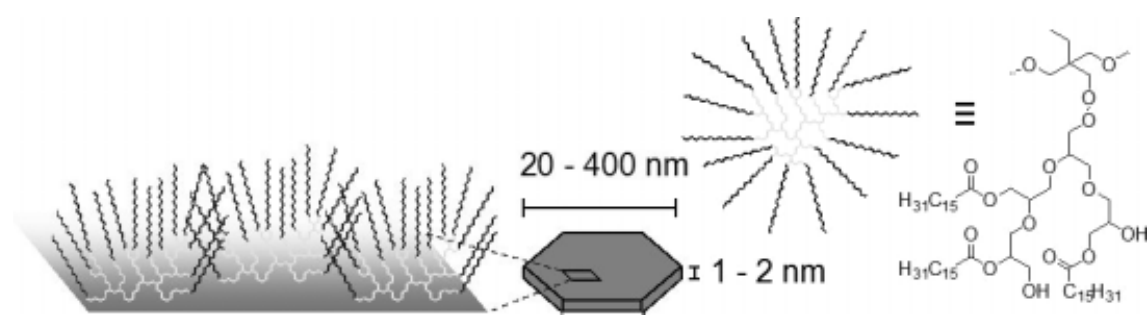

Figure 2. Proposed structure of palladium-platelet polymer hybrids. 


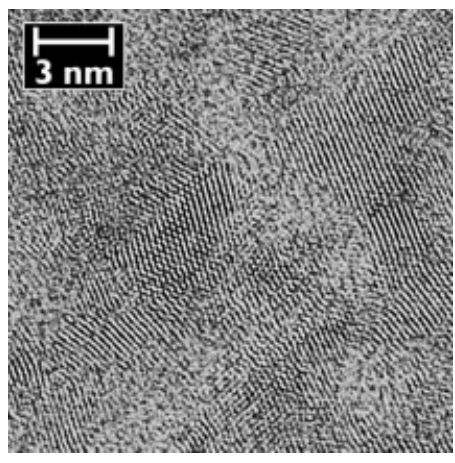

Figure 3. HRTEM image of a part of a hexagonal platelet with lattice plane fringes due to $1 / 3\{422\}$ reflections. Note that platelets have been fragmented under the conditions of the experiment.

served fringe spacing of $0.2361 \mathrm{~nm}$ deviates distinctly from the $\{111\}$ lattice plane spacing $(0.2246 \mathrm{~nm})$ of face centered cubic (fcc) palladium metal. Instead, it agrees fairly well with the spacing $(0.2382 \mathrm{~nm})$ of fringes due to kinematically forbidden $1 / 3\{422\}$ reflections expected to appear in thin films or platelets with $\{111\}$ surfaces and rather small thicknesses in the perpen dicular direction. ${ }^{[2]}$ The selected area electron diffraction pat tern (SAED) of a single platelet confirms this finding (Fig. 4). The observed pattern corresponds to a fcc palladium crystal viewed along a $<111>$ direction, i.e., the large faces of the hexa gon platelets correspond to $\{111\}$. Besides regular diffraction spots of (220) and (422) type, the SAED pattern is dominated by fractional (forbidden) $\{422\}$ spots, as indicated in Figure 4. From the diffraction data a lattice constant of $a=0.387 \mathrm{~nm}$ is calculated, which is in excellent agreement with literature data for palladium $(a=0.389 \mathrm{~nm}){ }^{[25]}$

Sample preparation for electron microscopy involves drying the colloidal solution on the surface of a carbon coated copper grid. Moreover, changes to the sample caused by the electron beam can occur. In the vast literature on colloidal metal parti cles, it is usually assumed, without further evidence, that the observed structures are not affected and resemble the solution

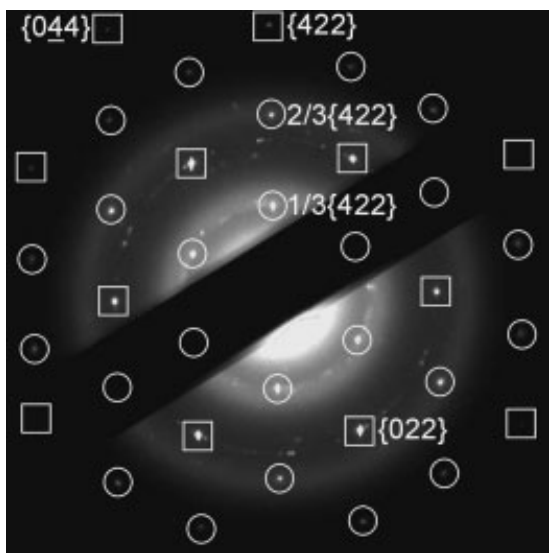

Figure 4. Selected area electron diffraction pattern of a single palladium platelet (prepared with PG6000-C15 10 ). Regular reflections are marked by open squares, forbidden reflections are marked by circles. structure. A conceivable formation of Pd nanoparticles only in the electron microscope, e.g., by reduction of traces of residual $\mathrm{Pd}^{\mathrm{II}}$ compounds, seems very unlikely. ${ }^{[26]}$ The observed platelets of regular shape, narrow size distribution and uniform arrange ment with their hexagonal faces parallel to the carbon support cannot be understood in terms of irradiation induced particle formation. Nonewithstanding, further studies by extended $\mathrm{X}$ ray absorption fine structure analysis (EXAFS) were carried out to provide confirmation. EXAFS enables the investigation of the particles directly in solution under mild conditions. The spectra confirm the particles to be composed of palladium(0). The spectra are identical to those of palladium foil, and within the accuracy of the experiment ( $>90 \%$ palladium( $(0))$ there is no indication of other oxidation states.

Ultrafiltration with solvent stable membranes is a conveni ent method for recovering a soluble catalyst after a catalytic re action, and also provides some preliminary insight into the shear sensitivity of the platelet dispersions. Catalytic hydro genation of cyclohexene was investigated as a model reaction. The catalytic ability of the platelets does not differ dramatically when compared to hydrogenation with spherical colloids stabi lized by the same polymers and under otherwise identical conditions, which is expected since hydrogenation is a rather non shape selective reaction. Recovery of the polyglycerol sta bilized platelets from the reaction solutions obtained from hydrogenation experiments by ultrafiltration did not result in any change in size or shape.

\section{Conclusions}

In summary, we report on the shape selective synthesis of palladium platelets stabilized by amphiphilic hyperbranched polymers. Very thin ( $<10$ atomic layers) hexagonal palladium platelets with sizes of fifteen to several hundred nanometers in the other two dimensions are obtained. Remarkably, despite their high aspect ratio, the platelets are also dimensionally stable under some degree of shear. Furthermore the system is exclusively based on readily available reagents.

\section{Experimental}

Carbon monoxide $(99.9 \%$ ) supplied by Gerling \& Holz and hydro gen $(99.999 \%)$ supplied by Messer Griesheim were used as received Toluene $(99.5 \%)$ supplied by Merck was used as received or freshly distilled from sodium under argon in the case of reduction with $\mathrm{LiBEt}_{3} \mathrm{H}$. Palladium acetate was supplied by Umicore via Merck; $\mathrm{LiBEt}_{3} \mathrm{H}$ supplied by Aldrich as a $1 \mathrm{M}$ solution in tetrahydrofuran was used as received. Hyperbranched polyglycerol and polyethyleneimine were supplied by Hyperpolymers GmbH [16].

Esterification [18] resp. amidation [11] was performed according to literature procedures. In a typical reduction procedure amphiphilic polyglycerol or polyethyleneimine respectively were dissolved together with $\mathrm{Pd}(\mathrm{OAc})_{2}$ in 3 to $20 \mathrm{~mL}$ of toluene (dry toluene in case of reduc tion with $\mathrm{LiBEt}_{3} \mathrm{H}$ ) in a Schlenk tube. In case of $\mathrm{H}_{2}$ or $\mathrm{CO}$ as reducing agent the gas was bubbled through the solution for three minutes be fore the flask was closed under reducing atmosphere, and stored at room temperature overnight. Reduction by the residual $\mathrm{OH}$ groups of the polyglycerol was carried out keeping a solution of polymer and 
$\mathrm{Pd}(\mathrm{OAc})_{2}$ at $60^{\circ} \mathrm{C}$ over night. Reduction with $\mathrm{LiBEt}_{3} \mathrm{H}$ occurred im mediately after adding $1 \mathrm{~mL}$ of dry toluene incorporating the desired amount of $\mathrm{LiBEt}_{3} \mathrm{H}$ to the solution of metal salt and polymer.

The diffusion of $\mathrm{CO}$ at room temperature from the gas phase into the solution is sufficiently fast, as shown by two otherwise identical ex periments where one flask was shaken overnight $(100 \mathrm{rpm})$ instead of simply storing it overnight (entry 15 , Table 1 ). The resulting diameters are identical within experimental errors.

TEM and SAED investigation were carried out on a LEO 912 Ome ga apparatus using an acceleration voltage of $120 \mathrm{kV}$. The particle sizes were determined by measuring the average diameters (average of larg est and smallest possible distance between two opposite points on the platelet edge) of ca. 100 platelets and performing a nonlinear least squares fit on the data to a Gaussian model. The center and the half width of the probability distribution function are given in Table 1.

HRTEM investigation was done using a JEM 4010 operating at $400 \mathrm{kV}$ accelerating voltage. Micrographs recorded at optimum imag ing conditions (Scherzer focus) were digitized for image processing. Diffractograms of HRTEM images obtained by Fourier transformation were used to measure lattice fringe spacings.

EXAFS Data Collection: Palladium K edge $(24350 \mathrm{eV})$ EXAFS spectra were measured at the Hamburger Synchrotronstrahlungslabor (Hasylab) Hamburg, Germany, Beamline X1, using a Si(111) double crystal monochromator. The monochromator was detuned to $50 \%$ in tensity to avoid effects of higher harmonics present in the $\mathrm{X}$ ray beam. The measurements were done in the transmission mode using opti mized ion chambers as detectors. To decrease noise scans were made in $k$ space and three scans were collected for each sample. Measurements were performed at room temperature.

EXAFS Data Analysis: A Pd foil was measured simultaneously with each sample. The energy was calibrated by assigning the first inflection point of the Pd foil to $24350 \mathrm{eV}$. Theoretical reference data were gen erated in the commercially available program XDAP [27] using FEFF8 [28]. The EXAFS data were analyzed using XDAP [27,29]. During R space fitting the difference file technique [27] was used in order to con clude a good analysis for all contributions in all weightings [30].

[1] a) J. S. Bradley, in Clusters and Colloids: From Theory to Applications (Ed: G. Schmid), VCH, Weinheim, Germany 1994, p. 459. b) Nano particles and Nanostructured Films (Ed: J. H. Fendler), Wiley VCH, Weinheim, Germany 1998. c) Active Metals: Preparation, Character ization, Applications (Ed: A. Fürstner), VCH, Weinheim, Germany 1996. d) H. Bönnemann, R. M. Richards, Eur. J. Inorg. Chem. 2001, 2455.

[2] a) M. Faraday, Philos. Trans. R. Soc. London 1857, 147, 145. b) W. Ostwald, Die Welt der Vernachlässigten Dimensionen, Steinkopff, Dresden, Germany 1915. c) L. D. Rampino, F. F. Nord, J. Am. Chem. Soc. 1941, 63, 2745.

[3] a) M. T. Reetz, W. Helbig, J. Am. Chem. Soc. 1994, 116, 7401. b) M. T. Reetz, M. Winter, R. Breinbauer, T. Thurn Albrecht, W. Vo gel, Chem. Eur. J. 2001, 7, 1084. c) G. Bredig, Angew. Chem. 1898, 10, 951. d) L. Rodríguez Sánchez, M. C. Blanco, M. A. López Quintela, J. Phys. Chem. B 2000, 104, 9683.

[4] a) P. H. Hess, P. H. Parker, Jr., J. Appl. Polym. Sci. 1966, 10, 1915. b) Y. N. C. Chan, R. R. Schrock, R. E. Cohen, Chem. Mater. 1992, 4, 24. c) A. Duteil, R. Quéau, B. Chaudret, R. Mazel, C. Roucau, J. S. Bradley, Chem. Mater. 1993, 5, 341. d) T. Ould Ely, C. Pan, C. Amiens, B. Chaudret, F. Dassenoy, P. Lecante, M. J. Casanove, A. Mosset, M. Respaud, J. M. Broto, J. Phys. Chem. B 2000, 104, 695. e) R. Choukroun, D. de Caro, B. Chaudret, P. Lecante, E. Snoeck, New J. Chem. 2001, 25, 525 .

[5] a) K. Fukaya, S. Ino, S. Ogawa, Trans. Jpn. Inst. Met. 1978, 19, 445. b) F. Robinson, M. Gillet, Thin Solid Films 1982, 98, 179. c) A. Re nou, A. Rudra, Surf. Sci. 1985, 156, 69. d) A. Renou, J. M. Penisson,
J. Cryst. Growth 1986, 78, 357. e) H. Hofmeister, Z. Phys. D 1991, 19 , 307.

[6] a) J. Turkevich, P. C. Stevenson, J. Hillier, Discuss. Faraday Soc. 1951, 11, 55. b) B. Brüche, Kolloid Z. 1960, 170, 97. c) W. O. Milligan, R. H. Moriss, J. Am. Chem. Soc. 1964, 86, 3461. d) J. Turkevich, G. Kim, Science 1970, 169, 873. e) A. C. Curtis, D. G. Duff, P. P. Edwards, D. A. Jefferson, B. F. G. Johnson, A. I. Kirkland, A. S. Wallace, $A n$ gew. Chem. Int. Ed. Engl. 1988, 27, 1530.

[7] a) M. Antonietti, E. Wenz, L. Bronstein, M. Seregina, Adv. Mater. 1995, 7, 1000. b) J. P. Spatz, A. Roescher, M. Moeller, Adv. Mater. 1996, 8, 337. c) M. Möller, J. P. Spatz, Curr. Opin. Colloid Interface Sci. 1997, 2, 177. d) J. Walter, Adv. Mater. 2000, 12, 31. e) J. S. Bradley, B. Tesche, W. Busser, M. Maase, M. T. Reetz, J. Am. Chem. Soc. 2000 , 122, 4631. f) X. Fu, Y. Wang, N. Wu, L. Gui, Y. Tang, Langmuir 2002 , 18, 4619. g) D. O. Yener, J. Sindel, C. A. Randall, J. H. Adair, Lang muir 2002, 18, 8692. h) H. Cölfen, Macromol. Rapid Commun. 2001, 22, 219. i) L. Addadi, S. Weiner, Angew. Chem. Int. Ed. Engl. 1992, 31,153 .

[8] a) Y. Sun, Y. Xia, Science 2002, 298, 2176. b) M. Maillard, S. Giorgio, M. P. Pileni, $A d v$. Mater. 2002, 14, 1084. c) I. Pastoriza Santos, L. M. Liz Marzan, Nano Lett. 2002, 2, 903. d) S. Chen, Z. Fan, D. L. Carroll, J. Phys. Chem. B 2002, 106, 10777. e) Y. Sun, Y. Xia, Adv. Mater. 2003, 15, 695. f) R. Jin, Y. C. Cao, E. Hao, G. S. Métraux, G. C. Schatz, C. A. Mirkin, Nature 2003, 425, 487.

[9] a) T. S. Ahmadi, Z. L. Wang, T. C. Green, A. Henglein, M. A. El Sayed, Science 1996, 272, 1924. b) H. P. Choo, K. Y. Liew, W. A. K. Mahmood, H. Liu, J. Mater. Chem. 2001, 11, 2906. c) A. Mayer, M. Antonietti, Colloid Polym. Sci. 1998, 276, 769. d) Y. Zhou, C. Y. Wang, Y. R. Zhu, Z. Y. Chen, Chem. Mater. 1999, 11, 2310.

[10] a) S. Mecking, R. Thomann, H. Frey, A. Sunder, Macromolecules 2000, 33, 3958. b) S. Mecking, U. Schlotterbeck, R. Thomann, M. Sod demann, M. Stieger, W. Richtering, H. Kautz, Polym. Mater. Sci. Eng. 2001, 84, 511.

[11] C. Aymonier, U. Schlotterbeck, L. Antonietti, P. Zacharias, R. Tho mann, J. C. Tiller, S. Mecking, Chem. Commun. 2002, 3018.

[12] R. Sablong, U. Schlotterbeck, D. Vogt, S. Mecking, Adv. Synth. Catal. $\mathbf{2 0 0 3}, 345,333$

[13] a) P. J. Flory, J. Am. Chem. Soc. 1952, 74, 2718. b) B. Voit, Acto Polym. 1995, 46, 87. c) Y. H. Kim, J. Polym. Sci., Part A: Polym. Chem. 1998, 36, 1685. d) A. Sunder, J. Heinemann, H. Frey, Chem. Eur. J. 2000, 6, 2499.

[14] Linear polymer: DB $0 \%$, calculated according to $\mathrm{DB}=2 \mathrm{D} /(2 D+L)$, $D=$ dendritic units (tertiary amine), $L=$ linear units (secondary amine), D. Hölter, A. Burgath, H. Frey, Acta Polym. 1997, 48, 30.

[15] a) M. Fischer, F. Vögtle, Angew. Chem. Int. Ed. 1999, 38, 885. b) A. W. Bosman, H. M. Janssen, E. W. Meijer, Chem. Rev. 1999, 99 , 1665. c) S. Hecht, J. M. J. Fréchet, Angew. Chem. Int. Ed. 2001, 40, 74. d) G. E. Oosterom, J. N. H. Reek, P. C. J. Kamer, P. W. N. M. van Leeuwen, Angew. Chem. Int. Ed. 2001, 40, 1828.

[16] Hyperpolymers GmbH, www.hyperpolymers.com

[17] Product information brochure Lupasol, BASF AG.

[18] a) A. Sunder, M. Krämer, R. Hanselmann, R. Mülhaupt, H. Frey, Angew. Chem. Int. Ed. 1999, 38, 3552. b) S. E. Stiriba, H. Kautz, H. Frey, J. Am. Chem. Soc. 2002, 124, 9698.

[19] J. Decker, B. Geisler, R. Paciello, N. Mahr, H. Vos, E. Zeller, M. Rö per, WO99/36382, 1999.

[20] V. M. Garamus, T. Maksimova, W. Richtering, C. Aymonier, R. Tho mann, L. Antonietti, S. Mecking, Macromolecules, in press.

[21] Corresponding to complete conversion of all $\mathrm{NH}_{2}$ groups. $100 \%$ degree of amidation corresponds to complete conversion of $\mathrm{R}_{2} \mathrm{NH}$ and $\mathrm{RNH}_{2}$ to $\mathrm{R}_{2} \mathrm{NC}(=\mathrm{O}) \mathrm{C}_{15} \mathrm{H}_{31}$ resp. $\mathrm{RNHC}(=\mathrm{O}) \mathrm{C}_{15} \mathrm{H}_{31}$. The PEI utilized contains a ratio of tertiary amine (branching, dendritic units): secondary amine (linear units): primary units (ends of branches) of ca. 30/40/30 according to ${ }^{13} \mathrm{C}$ NMR, in accordance with literature reports: D. N. Roark, B. C. McKusick, in Ullmann's Encyclopedia of Industrial Chemistry, Vol. A3, 5th ed. (Ed: W. Gerhartz), VCH, Weinheim, Ger many 1985 , p. 239. 
[22] L. Qi, H. Cölfen, M. Antonietti, Nano Lett. 2001, 1, 61.

[23] a) H. Hirai, Y. Nakao, N. Toshima, J. Macromol. Sci., Chem. 1979, 13, 727. b) E. Bayer, W. Schumann, J. Chem Soc., Chem. Commun. 1986, 949. c) J. S. Bradley, E. W. Hill, S. Behal, C. Klein, B. Chaudret, A. Duteil, Chem. Mater. 1992, 4, 1234. d) A. Borsla, A. M. Wilhelm, H. Delmas, Catal. Today 2001, 66, 389. e) I. Pstoriza Santos, L. M. Liz Marzán, Langmuir 2002, 18, 2888. f) H. P. Choo, K. Y. Liew, H. Liu, J. Mater. Chem. 2002, 12, 934.

[24] a) G. Nihoul, K. Abdelmoula, J. J. Metois, Ultramicroscopy, 1984, 12, 353. b) Y. Golan, L. Margulis, G. Hodes, I. Rubinstein, J. L. Hutchi son, Surf. Sci. 1994, 311, L633.

[25] Crystal Data Determinative Tables, Vol. II, 3rd ed. (Eds: J. D. H. Donnay, H. M. Ondik), US Department of Commerce, National Bu reau of Standards, Washington, DC 1973.
[26] Investigations of the samples with respect to the metal oxidation state by XPS were unsuccessful, presumably due to an insufficient penetra tion depth into the polymer layer under the experimental conditions. We thank Andreas Häfele for carrying out these experiments at the Freiburg Materials Research Center.

[27] M. Vaarkamp, J. C. Linders, D. C. Koningsberger, Phys. B. 1995, 208 209, 159.

[28] A. L. Ankudinov, B. Ravle, J. J. Rehr, S. D. Conradson, Phys. Rev. B 1998, 7565 .

[29] D. C. Koningsberger, B. L. Mojet, G. E van Dorssen, D. E. Ramaker, Top. Catal. 2000, 10, 143.

[30] M. Tromp, J. A. van Bokhoven, A. M. Arink, J. H. Bitter, G. van Ko ten, D. C. Koningberger, Chem. Eur. J. 2002, 8, 5667. 